\title{
Commentary: Indexing the nutritional status of cardiac surgery patients: proof is in the pudding
}

\author{
Siamak Mohammadi, MD, FRCSC, and Dimitri Kalavrouziotis, MD, FRCSC
}

From the Department of Cardiac Surgery, Quebec Heart and Lung Institute, Quebec City, Quebec, Canada. Disclosures: Authors have nothing to disclose with regard to commercial support.

Received for publication Aug 25, 2019; accepted for publication Aug 26, 2019; available ahead of print Sept 20, 2019

Address for reprints: Siamak Mohammadi, MD, FRCSC, Department of Cardiac Surgery, Quebec Heart and Lung Institute, 2725 Chemin Sainte-Foy, Quebec City, Quebec, Canada, G1V 4G5 (E-mail: siamak.mohammadi@ fmed.ulaval.ca).

J Thorac Cardiovasc Surg 2020;160:154-5

$0022-5223 / \$ 36.00$

Copyright $(2019$ by The American Association for Thoracic Surgery

https://doi.org/10.1016/j.jtcvs.2019.08.097

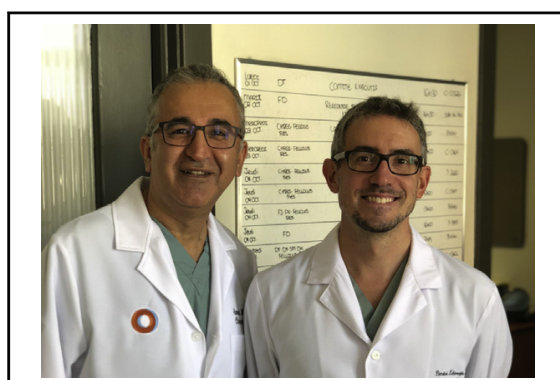

Siamak Mohammadi, MD, FRCSC, and Dimitri Kalavrouziotis, MD, FRCSC

\section{Central Message}

The prognostic nutritional index is easily obtained using routine bloodwork before cardiac surgery and may provide a useful tool for identifying malnourished patients and improving outcomes.

See Article page 145 preoperative nutritional status and (2) the prediction of early and late clinical outcomes.

In this issue of the Journal, Lee and colleagues ${ }^{5}$ sought to evaluate the predictive value of the prognostic nutritional index (PNI) as a preoperative risk stratification tool among 347 adult patients undergoing cardiac surgery with cardiopulmonary bypass, in a single-center pilot study. The PNI was easily obtained using the preoperative serum albumin value and total lymphocyte count; a cut-off value of 46.13 was identified a priori below which a state of malnutrition was present. Not surprisingly, the typical malnourished patient was elderly, with a lower body mass index, renal failure, and previous cardiac surgery. In multivariate analysis, the independent risk factors associated with the combined end-point of early mortality and adverse events were (1) lower PNI; (2) advanced age; and (3) longer cardiopulmonary bypass and crossclamp times. However, lower PNI did not predict mid-term outcomes, including mortality and major cardiovascular events using Kaplan-Meier analysis, despite a greater risk profile of patients with low PNI.

The authors should be congratulated on their efforts to evaluate the seldom-studied issue of preoperative nutritional status among patients undergoing cardiac surgery. The simplicity of calculating the PNI based on routine preoperative serum biochemistry and complete blood counts makes it an attractive and easily reproducible tool for perioperative risk stratification compared with the plethora of existing nutritional assessment measures. Moreover, the

need to administer a questionnaire with its inherent subjectivity is also eliminated. The data published by Lee and colleagues $^{5}$ suggest that malnutrition is highly prevalent in patients undergoing cardiac surgery (up to $26.7 \%$ of their cohort), and its relationship with postoperative mortality and adverse events suggests that nutritional status cannot be ignored as major prognostic factor in cardiac surgery.

However, a word of caution is advisable when interpreting the results of the study. The retrospective nature of the study, the use of a combined end-point including 9 events that differ vastly with respect to their clinical significance, a significant attrition of patients after 2 years of follow-up with a complete lack of longitudinal data in $10 \%$ of the cohort, and the absence of validation using an external patient population all make the drawing of solid conclusions problematic. A potential pathophysiologic relationship between a lower PNI and a greater incidence of early complications was not explored. Furthermore, multiple unadjusted subgroup analyses to identify those patients who may benefit the most from using PNI as a nutritional screening tool were not convincing and could be subject to type II error due to small patient numbers.

The real missing piece of the puzzle in the present study ${ }^{5}$ is the absence of data on the efficacy of perioperative nutritional interventions targeting malnourished patients in an effort to optimize perioperative nutritional status and outcomes following cardiac surgery. The clinical impact of 
such interventions continues to elude us. A prospective comparative study is needed to evaluate the predictive utility of PNI as a reliable screening tool before cardiac surgery and to remove residual doubts of the PNI as a simple "innocent bystander" among high-risk patients whose prognosis is already sealed. If preoperative nutritional support can be shown to decrease postoperative complications, a true cause-effect relationship between nutritional status and outcomes may exist; the study's current design does not allow the reader to answer this question. Despite these shortcomings, the study by Lee and collegues ${ }^{5}$ sheds some light on a novel aspect of perioperative care in which the early detection of malnutrition can serve as a trigger for the initiation of comprehensive nutritional assessment and support to further improve the early and late outcomes of cardiac surgical patients.

\section{References}

1. Okada S, Shimada J, Daishiro K, Tsunezuka H, Teramukai S, Inoue M. Clinical significance of prognostic nutritional index after surgical treatment in lung cancer. Ann Thorac Surg. 2017;104:296-302.

2. Arends J, Baracos V, Bertz H, Bozzetti F, Duetz NEP, Erickson N, et al. ESPEN expert group recommendations for action against cancer-related malnutrition. Clin Nutr. 2017;36:1187-96.

3. Almeida AI, Correia M, Camilo M, Ravasco P. Nutritional risk screening in surgery: valid, feasible, easy. Clin Nutr. 2012;31:206-11.

4. Bauer J, Kaiser M, Sieber C. Evaluation of nutritional status in older persons: nutritional screening and assessment. Curr Opin Clin Nutr Metab Care. 2010;13:8-13.

5. Lee SI, Ko KP, Choi CH, Park CH, Park KY, Son KH. Does the prognostic nutritional index have a predictive role in the outcomes of adult cardiac surgery? J Thorac Cardiovasc Surg. 2020;160:145-53.e3. 